\title{
A HYBRID CONTROL SCHEME FOR FREEWAY SYSTEMS
}

\author{
Simona Sacone $^{*}$ Elisa Franco ${ }^{* *}$ Thomas Parisini $^{* *}$ \\ * Dept. of Communications, Computer and Systems Sciences \\ DIST-University of Genova, Italy \\ ** Dept. of Electrical, Electronic and Computer Engineering \\ DEEI-University of Trieste, Italy
}

\begin{abstract}
The development of a hybrid control scheme for freeway systems is the objective of the paper. A finite number of models is defined, each relevant to a specific traffic condition. The system state variables are the differences between the traffic density and the traffic mean speed in each section and their desired values. A receding-horizon $(\mathrm{RH})$ regulator is defined for each model being the control variables the traffic volumes at onramps. A hybrid control scheme is proposed, which is composed of two control levels. The first control level consists of a finite class of models and RH control functions. The second level acts as a supervisor that chooses the best model and control law to be applied to the plant according to the present system state and possible external events. The application of the proposed control scheme makes it possible to guarantee some suitable stability properties of the origin as an equilibrium point of the system considered at the first control level. Copyright (C) 2005 IFAC
\end{abstract}

Keywords: Road traffic, nonlinear models, switched control, stability analysis.

\section{INTRODUCTION}

An effective management of freeways and interurban roadways is definitely a crucial aspect in our daily life. Such systems are complex large-scale systems originally conceived so as to provide virtually unlimited mobility to users. As a matter of fact, the available infrastructure capacity is not in most cases sufficient (and it cannot be always increased) leading to the the occurrence of both recurrent and non-recurrent congestion phenomena. This has soon highlighted that what is necessary is an efficient utilization of the available road capacity by designing suitable modelling, optimization and control methods.

Freeway systems have such peculiar characteristics making it necessary to define dedicated models and control actions. In the literature, many freeway control methods have been proposed mainly based on the macroscopic modelling theory. In this context, a widespread control measure adopted for freeway stretches refers to the so-called ramp metering control. The control variable characterizing such a kind of control action is the traffic volume on the on-ramps which is in some way modulated regulating the flow of incoming traffic to the freeway stretch.

Ramp metering has been extensively studied starting from very simple local fixed-time ramp metering strategies (Wattleworth, 1967), to closed-loop local strategies (Papageorgiou et al., 1991), to multivariable regulator approaches (Isaksen and Payne, 1973), (Papageorgiou et al., 1990b), to arrive to nonlinear optimal ramp metering strategies (Zhang et al., 1996), (Kotsialos et al., 2002), (Di Febbraro et al., 2001).

A significant feature of freeway systems that still deserves major research efforts is that different traffic control problems should be stated and solved for the same road stretch, depending on traffic conditions (from regular traffic to severe congestion phenomena) and exceptional situations (critical weather conditions, accidents, etc.). This aspect will be the key aspect of this work in which the freeway system will be separately modelled and controlled under different traffic conditions and considered in a control scheme as the one developed in (Parisini and Sacone, 1999), (Parisini and Sacone, 2001). The proposed scheme is a hybrid control scheme for nonlinear discrete- 
time systems composed of two control levels. The first control level consists of a finite class of models and receding-horizon nonlinear control functions. The second level acts as a supervisor that chooses from the set defined at the first level the best pair model/control law to be applied to the plant according to the present system state.

\section{THE NONLINEAR MACROSCOPIC MODEL}

The adopted macroscopic model was first proposed by Payne in (Payne, 1971) (see also (Papageorgiou et al., 1990 a)). Payne's model is based on the discretization (in both space and time) of the conservation equation, from which the following equation is derived

$$
\begin{aligned}
& \rho_{j}(k+1)=\rho_{j}(k)+ \\
& +\frac{T}{\Delta_{j}}\left(q_{j-1}(k)-q_{j}(k)+r_{j}(k)-s_{j}(k)\right), \\
& \quad j=1, \ldots, S ; k=0,1, \ldots, K \quad(1)
\end{aligned}
$$

where $k$ denotes the control temporal stage and $j$ denotes one of the sections into which the freeway stretch has been divided. $K$ temporal stages and $S$ sections (of length $\Delta_{j}$ varying approximately from 500 to $1000 \mathrm{~m}$ ) have been considered. $T$ is the sample time interval, $\rho_{j}(k)$ is the traffic density (number of vehicles per length unit in section $j$ at time $k T), q_{j}(k)$ is the traffic volume (number of vehicles per time unit leaving section $j$ during the time interval $[k T,(k+$ 1)T]), and $r_{j}(k)$ and $s_{j}(k)$ are the on-ramp and off-ramp traffic volumes for section $j$, respectively. Actually, $r_{j}(k)$ and $s_{j}(k)$ are different from zero only for the sections that contain on/off ramps. The indices of such sections make up the set $\mathcal{I}_{r}$. Quantities $r_{j}(k)$ play the role of control variables; such variables will be defined as nonnegative quantities and will be bounded from above by the traffic demands present at the corresponding on-ramps.

The third aggregate variable of interest is the mean traffic speed, $v_{j}(k)$, whose dynamics is described by the equation

$$
\begin{aligned}
v_{j}(k+1) & =v_{j}(k)+\frac{T}{\tau}\left[V\left(\rho_{j}(k)\right)-v_{j}(k)\right]+ \\
+ & \frac{T}{\Delta_{j}} v_{j}(k)\left(v_{j-1}(k)-v_{j}(k)\right)+ \\
& -\frac{\mu T\left(\rho_{j+1}(k)-\rho_{j}(k)\right)}{\tau \Delta_{j}\left(\rho_{j}(k)+\chi\right)} \\
& j=1, \ldots, S ; k=0,1, \ldots, K
\end{aligned}
$$

where $\tau, \mu$, and $\chi$ are parameters to be determined experimentally (by a suitable identification procedure). $V\left(\rho_{j}(k)\right)$ is the steady-state speed-density characteristic, which, in major classical models, takes on the form

$$
V\left(\rho_{j}(k)\right)=V_{f}\left[1-\left(\rho_{j}(k) / \rho_{\max }\right)^{l}\right]^{m}
$$

where $V_{f}$ denotes the "free" speed (a value experimentally determined on the basis of real traffic data and very low values of traffic density), $\rho_{\max }$ is the so-called "jam density", and $l$ and $m$ are real-valued positive parameters. In the model here proposed, the form of the steady-state speed-density characteristic is significantly simplified to become a piecewise constant function $\tilde{V}\left(\rho_{j}(k)\right)$. This approximation, which can give rise to effective models when applied to real case studies (Magliolo and Sacone, 2004), is depicted in Fig. 1 for a generic set of parameters. The three

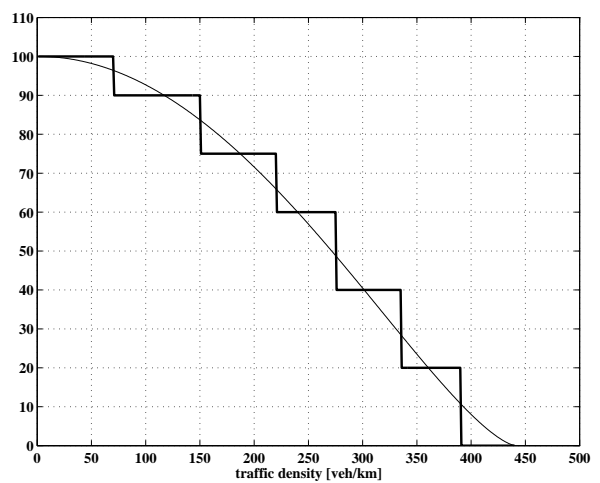

Fig. 1. The classical form of function $V\left(\rho_{j}(k)\right)$ and its approximation $\tilde{V}\left(\rho_{j}(k)\right)$ (thick line)

aggregate state variables so far defined are related to one another by the following weighted sum

$$
\begin{gathered}
q_{j}(k)=\alpha \rho_{j}(k) v_{j}(k)+(1-\alpha) \rho_{j+1}(k) v_{j+1}(k), \\
j=1, \ldots, S-1 ; k=0,1, \ldots, K
\end{gathered}
$$

where $0 \leq \alpha \leq 1$ is an appropriate weighting factor (which again needs to be identified on the basis of real traffic data). The off-ramp volumes $s_{j}(k)$ are related to the traffic volumes $q_{j-1}(k)$ through the relationship (Papageorgiou and Mayr, 1982) $s_{j}(k)=$ $\gamma_{j} q_{j-1}(k), j \in \mathcal{I}_{r}$, where $0<\gamma_{j}<1$. Substituting this relation and (4) into (1) yields

$$
\begin{aligned}
& \rho_{j}(k+1)=\rho_{j}(k)+\frac{T}{\Delta_{j}}\left[\alpha\left(1-\gamma_{j}\right) \rho_{j-1}(k) v_{j-1}(k)+\right. \\
& +\left(1-2 \alpha+\gamma_{j} \alpha-\gamma_{j}\right) \rho_{j}(k) v_{j}(k)+ \\
& \left.\quad-(1-\alpha) \rho_{j+1}(k) v_{j+1}(k)+r_{j}(k)\right] \\
& \quad j=1, \ldots, S ; k=0,1, \ldots, K
\end{aligned}
$$

Equations (2) and (5) should be modified (in a simple way) for both the first and the last sections of the freeway.

As already mentioned, the goal of our control scheme is that of maintaining, under different traffic conditions, the freeway system working under desired values of the traffic densities and traffic mean speeds. As already done, for instance in (Chien et al., 1997), it is possible to define suitable values for the two state variables in each section. In this work, several operating conditions for the considered freeway system have been identified with reference to the above introduced piecewise constant approximation of the steady-state speed-density relationship. Specifically, it is supposed that, on the basis of real data, an effective approximation of the steady-state speed-density characteristic can be found. This allows to identify 
the values $\bar{\rho}_{i}, i=1, \ldots, N$ of the traffic densities corresponding to the $N$ discontinuities of the defined approximated function and to find the corresponding constant values $\bar{V}_{i}, i=1, \ldots, N$ of the same function. More precisely, the approximation of $V\left(\rho_{j}(k)\right)$ can be expressed as

$$
\begin{aligned}
\tilde{V}\left(\rho_{j}(k)\right) \triangleq & \bar{V}_{i} \\
& \bar{\rho}_{i} \leq \rho_{j}(k) \leq \bar{\rho}_{i+1}, i=1, \ldots, N
\end{aligned}
$$

where $\bar{V}_{1}=V_{f}, \bar{V}_{N}=0, \bar{\rho}_{1}=0$ and $\bar{\rho}_{N+1}=\rho_{\max }$.

For the sake of simplicity, a control scheme for a small area including few freeway sections is considered. In such a scheme it can be supposed that the freeway portion is made up of very similar sections (characterized by the same parameter set and by the same traffic fundamental diagram). Then, the supervision system can identify $N$ different operating conditions of traffic in the freeway portion and define the corresponding $N$ desired values of the traffic density and of the traffic mean speed in each section. In doing this, it is now supposed that the desired values of the system aggregate variables are the same for each section.

Let us now introduce, the set $\left\{\left(\tilde{\rho}_{i}, \tilde{v}_{i}\right), i=1, \ldots, N\right\}$, where $\tilde{\rho}_{i}: \bar{\rho}_{i} \leq \tilde{\rho}_{i} \leq \bar{\rho}_{i+1}$ and $\tilde{v}_{i}=\bar{V}_{i}$ are the desired values of the traffic density and of the traffic mean speed in the $i$-th traffic configuration. It can be noted that the desired state $\left(\tilde{\rho}_{i}, \tilde{v}_{i}\right), \forall i$ must also be an equilibrium point of the second-order Payne model here adopted. To this end, the values $\tilde{r}_{i} \triangleq \gamma \tilde{\rho}_{i} \tilde{v}_{i}, i=1, \ldots, N$ are defined (note that $\left.\gamma_{j}=\gamma, \forall j=1, \ldots, S\right)$. The traffic volumes at the on-ramps in the $i$-th traffic configuration are now expressed as $r_{j}(k)=\tilde{r}_{i}+\theta_{j}(k)$, where the control variables are now quantities $\theta_{j}(k)$ (the determination of such quantities must still guarantee that the traffic volumes at the on-ramps are nonnegative and bounded from above by the traffic demands at the corresponding on-ramps).

By considering the system working under the generic $i$-th traffic condition, tracking errors for the traffic density and traffic mean speed can be defined as

$$
\begin{aligned}
& \xi_{j}(k) \triangleq \rho_{j}(k)-\tilde{\rho}_{i} \\
& \nu_{j}(k) \triangleq v_{j}(k)-\tilde{v}_{i}
\end{aligned}
$$

whose dynamics, by using (5) can be written as

$$
\begin{aligned}
& \xi_{j}(k+1)=\rho_{j}(k+1)-\tilde{\rho}_{i}= \\
& \rho_{j}(k)+\frac{T}{\Delta_{j}}\left[\alpha\left(1-\gamma_{j}\right) \rho_{j-1}(k) v_{j-1}(k)+\right. \\
& +\left(1-2 \alpha+\gamma_{j} \alpha-\gamma_{j}\right) \rho_{j}(k) v_{j}(k)+ \\
& \left.\quad-(1-\alpha) \rho_{j+1}(k) v_{j+1}(k)+r_{j}(k)\right]-\tilde{\rho}_{i}= \\
& \quad \xi_{j}(k)+ \\
& \quad+\frac{T}{\Delta_{j}}\left[c_{j}^{1}\left(\xi_{j-1}(k)+\tilde{\rho}_{i}\right)\left(\nu_{j-1}(k)+\tilde{v}_{i}\right)+\right. \\
& +c_{j}^{2}\left(\xi_{j}(k)+\tilde{\rho}_{i}\right)\left(\nu_{j}(k)+\tilde{v}_{i}\right)+ \\
& \left.\quad-c_{j}^{3}\left(\xi_{j+1}(k)+\tilde{\rho}_{i}\right)\left(\nu_{j+1}(k)+\tilde{v}_{i}\right)+\tilde{r}_{i}+\theta_{j}(k)\right] \\
& \quad j=1, \ldots, S ; k=0,1, \ldots, K
\end{aligned}
$$

where $c_{j}^{1}=\alpha\left(1-\gamma_{j}\right), c_{j}^{2}=\left(1-2 \alpha+\gamma_{j} \alpha-\gamma_{j}\right)$, $c_{j}^{3}=(1-\alpha)$. By using $(2)$, it holds

$$
\begin{aligned}
\nu_{j}(k+1) & =v_{j}(k+1)-\tilde{v}_{i}= \\
& v_{j}(k)+\frac{T}{\tau}\left[\tilde{V}\left(\rho_{j}(k)\right)-v_{j}(k)\right]+ \\
& +\frac{T}{\Delta_{j}} v_{j}(k)\left(v_{j-1}(k)-v_{j}(k)\right)+ \\
& -\frac{\mu T\left(\rho_{j+1}(k)-\rho_{j}(k)\right)}{\tau \Delta_{j}\left(\rho_{j}(k)+\chi\right)}= \\
\nu_{j}(k)+ & \\
& \frac{T}{\tau}\left[\tilde{V}\left(\xi_{j}(k)+\tilde{\rho}_{i}\right)-\left(\nu_{j}(k)+\tilde{v}_{i}\right)\right]+ \\
& +\frac{T}{\Delta_{j}}\left(\nu_{j}(k)+\tilde{v}_{i}\right)\left(\nu_{j-1}(k)-\nu_{j}(k)\right)+ \\
& -\frac{\mu T\left(\xi_{j+1}(k)-\xi_{j}(k)\right)}{\tau \Delta_{j}\left(\xi_{j}(k)+\tilde{\rho}_{i}+\chi\right)} \\
& j=1, \ldots, S ; k=0,1, \ldots, K
\end{aligned}
$$

Equations (9) and (10) constitute a model describing the tracking errors dynamics. In a more compact form, it is possible to define $x(k) \triangleq \operatorname{col}(\underline{\xi}(k), \underline{\nu}(k)))$ with $\underline{\xi}(k) \triangleq \operatorname{col}\left(\xi_{j}(k), j=1, \ldots, S\right)$ and $\underline{\nu}(k) \triangleq$ $\operatorname{col}\left(\nu_{j}(k), j=1, \ldots, S\right)$. In the same way, we define the control vector as $u(k) \triangleq \operatorname{col}(\underline{\theta}(k))$, where $\underline{\theta}(k) \triangleq \operatorname{col}\left(\theta_{j}(k), j \in \mathcal{I}_{r}\right)$. Then, we can write (9) and (10) in the form of state equations

$$
\begin{aligned}
x(k+1) & =f^{i}(x(k), u(k)) \\
& i=1, \ldots, N ; k=0,1, \ldots, K-1
\end{aligned}
$$

Note that the origin is an equilibrium point of each nonlinear discrete-time system represented with (11).

\section{THE SWITCHING CONTROL SCHEME}

The proposed switching control scheme is here briefly outlined; the reader is addressed to (Parisini and Sacone, 2001), (Franco et al., 2004b) for further details. Consider a nonlinear discrete-time dynamic system described by:

$$
x(k+1)=f(x(k), u(k)), \quad t=0,1, \ldots
$$

where $x(k) \in \mathbb{R}^{n}$ and $u(k) \in \mathbb{R}^{m}$ are the state and control vectors respectively. At any time instant $k, f(x(k), u(k))=f^{i}(x(k), u(k))$, where $f^{i}$ is a dynamic sub-model and $x(k) \in \mathcal{X}^{i}$, with $\mathcal{X}^{i} \subset$ $\mathbb{R}^{n}$ denoting the state-space sub-domain where $f^{i}$ is considered as a valid model for the system. We assume that the sets $\mathcal{X}^{i}$ belong to class $\mathcal{Z}=\bigcup_{j=1}^{\infty} \mathcal{Z}_{j}, \mathcal{Z}_{j} \subset$ $\mathbb{R}^{q}$, where $\mathcal{Z}_{j}$ is a compact set containing the origin as an internal point. We also assume that $f^{i}$ belongs to a given class of sub-models, that is, $f^{i} \in \mathcal{F} \triangleq$ $\left\{f^{1}, \ldots, f^{N}\right\}$, with $f^{i} \in \mathcal{C}^{1}\left[\mathbb{R}^{n} \times \mathbb{R}^{m}, \mathbb{R}^{n}\right]$, and $f^{i}(0,0)=0$.

Moreover, $u(k)=\gamma^{i r}(x(k))$, where $\gamma^{i r}$ belongs to a finite class of control functions associated with submodel $f^{i}$, that is, $\gamma^{i r} \in \Gamma_{i} \triangleq\left\{\gamma^{i 1}, \ldots, \gamma^{i M_{i}}\right\}$. For the sake of notational convenience, let us introduce the sets $I \triangleq\{1, \ldots, N\}, \quad J^{i} \triangleq\left\{1, \ldots, M_{i}\right\}, i \in I$, 
characterizing the indexes associated with the class $\mathcal{F}$ of dynamic sub-models $f^{i}$ and the classes $\Gamma_{i}$ of control functions $\gamma^{i r}$ associated with model $f^{i}$, respectively. Then, the class of control functions can be written as $\Gamma_{i}=\left\{\gamma^{i r}: \mathcal{X}^{i} \mapsto \mathbb{R}^{m}, r \in J_{i}\right\}$.

In the considered switched system, both the active model and the applied control function can be changed in specific time instants, denoted as switching instants. More specifically, we assume that switching is controlled by a supervision system, which means that in the hybrid control scheme there is no autonomous jump from a pair $\left(f^{i}, \gamma^{i r}\right)$ to another one. In the considered case study, this means that the supervisor on the basis of the traffic conditions in the overall freeway portion, decides the kind of model and control action to be adopted. The structure and dynamic behavior of the supervisor are not matter of the present paper (more details can be found in (Parisini and Sacone, 1999),(Parisini and Sacone, 2001) and the references cited therein, where the supervision system is modelled by discrete-event methodologies).

Some important definitions still need to be introduced since they will be used later on in the application of the hybrid control scheme to freeway systems.

Definition 3.1. A switching law $\mathcal{S}$ associated with system (12) is a decision law that, at a generic timeinstant $\tau \in \mathbb{Z}$, identifies a subset of admissible pairs $\left(f^{i}, \gamma^{i r}\right)$ that it is possible to activate in order to satisfy a given property.

Accordingly, it is possible to define a switching sequence as a set collecting switching instants; formally, a switching sequence is denoted as

$$
\Xi \triangleq\left\{\tau_{0}, \tau_{1}, \ldots, \tau_{n}, \ldots\right\}, \quad \tau_{n} \in \mathbb{Z}^{+}, n \in \mathbb{N}
$$

Moreover, in the considered control scheme, the control functions $\gamma^{i r}$ are RH nonlinear control laws. A detailed statement of the RH control problem referred to system (12), for each pair $\left(f^{i}, \gamma^{i r}\right)$, can be found in (Parisini and Sacone, 2001), and here it will only be briefly recalled for the sake of completeness.

Problem 1. For any $i \in I$ and for any $r \in J_{i}$, find the RH optimal control law

$$
u^{R H_{o}^{i r}}(k)=\gamma^{R H_{o}^{i r}}(x(k)) \in \mathbb{R}^{m}
$$

where $u^{R H_{o}^{i r}}(k)$ is the first vector of the control sequence $u^{F H_{o}^{i r}}(k), \ldots, u^{F H_{o}^{i r}}\left(k+N^{i r}-1\right)$ that minimizes the finite horizon (FH) cost function:

$$
\begin{aligned}
& J_{F H}^{i r}\left(x(k), u(k), \ldots, u\left(k+N^{i r}-1\right), N^{i r}, a^{i r}, P^{i r}\right)= \\
& \sum_{n=k}^{k+N^{i r}-1} h^{i r}(x(n), u(n))+a^{i r}\left\|x\left(k+N^{i r}\right)\right\|_{P^{i r}}
\end{aligned}
$$

for the state $x(k) \in \mathcal{X}^{i} . N^{i r}$ is a positive integer denoting the length of the control horizon, $a^{i r} \in \mathbb{R}$ is a positive scalar and $P^{i r}$ is a positive-definite symmetric matrix.
When the optimal sequence is found, the optimal solution is

$$
\begin{aligned}
& J^{F H_{o}^{i r}}\left(x(k), N^{i r}, a^{i r}, P^{i r}\right)= \\
& \sum_{n=k}^{k+N^{i r}-1} h^{i r}\left(x^{F H_{o}^{i r}}(n), u^{F H_{o}^{i r}}(n)\right)+ \\
& \quad+a^{i r}\left\|x^{F H_{o}^{i r}}\left(k+N^{i r}\right)\right\|_{P^{i r}},
\end{aligned}
$$

where $x^{F H_{o}^{i r}}(n+1)=f^{i}\left(x^{F H_{o}^{i r}}(n), u^{F H_{o}^{i r}}(n)\right)$, and $n=k, \ldots, k+N^{i r}-1, x^{F H_{o}^{i r}}(k)=x(k)$. For simplicity, from now on we let $\gamma^{i r} \triangleq \gamma^{R H_{o}^{i r}}$. It is also worth noting that Problem 1 can be solved (in approximate way) on-line or off-line, depending on the specific context: this topic is discussed in previous works such as (Parisini and Sacone, 2001), (Parisini and Zoppoli, 1995).

If the parameters $N^{i r}, P^{i r}, a^{i r}$ in the FH cost functions (13) are suitably chosen, the control laws to be applied to the active sub-model $f^{i}$ have some very important stabilizing properties (see, for instance, (Keerthi and Gilbert, 1988), (Parisini and Zoppoli, 1995), (Parisini et al., 1998)). In particular, under suitable assumptions and modifications of the proofs, the RH control laws are characterized by the following properties:

1) The origin is a globally asymptotically stable equilibrium point of the closed-loop control system under the action of the RH optimal control law.

2) Consider the largest positive scalar $\bar{\theta}^{i r} \in \mathbb{R}^{+}$ such that $\mathcal{X}^{i r}\left(N^{i r}, a^{i r}, P^{i r}, \bar{\theta}^{i r}\right) \subset \mathcal{X}^{i}$, where the set $\mathcal{X}^{i r}\left(N^{i r}, a^{i r}, P^{i r}, \theta^{i r}\right)$ is defined as

$$
\begin{aligned}
& \mathcal{X}^{i r}\left(N^{i r}, a^{i r}, P^{i r}, \theta^{i r}\right) \triangleq \\
& \left\{x \in \mathcal{X}^{i}: J^{F H_{o}^{i r}}\left(x, N^{i r}, a^{i r}, P^{i r}\right) \leq \theta^{i r}\right\}
\end{aligned}
$$

The resulting set $\mathcal{X}^{i r}\left(N^{i r}, a^{i r}, P^{i r}, \bar{\theta}^{i r}\right)$ is an invariant set and a domain of attraction for the origin and the optimal cost function is a Lyapunov function for the pair $\left(f^{i}, \gamma^{i r}\right)$.

In the stability analysis of the overall control scheme, the Lyapunov functions $J^{F H_{o}^{i r}}\left(x, N^{i r}, a^{i r}, P^{i r}\right)$ and the invariant sets $\mathcal{X}^{i r}\left(N^{i r}, a^{i r}, P^{i r}, \bar{\theta}^{i r}\right)$ are exploited frequently. In this respect, we let

$$
\mathcal{V}^{i r}(x(k)) \triangleq J^{F H_{o}^{i r}}\left(x(k), N^{i r}, a^{i r}, P^{i r}\right)
$$

\section{APPLICATION OF THE HYBRID CONTROL SCHEME TO FREEWAY SYSTEMS}

The possibility of choosing among a finite set of models and regulators the best to be applied to each freeway section under the present traffic conditions is the main advantage of the proposed approach. Actually, it is easy to verify that the models described in Section 2 by equations (9) and (10) satisfy the assumption made in Section 3 for the definition of the hybrid control scheme. It is still necessary to define the class of regulators adopted for the case under concern. 
As a matter of fact, the behavior of traffic in a freeway section needs to be regulated in different ways dependent on the current traffic conditions. More specifically, $N$ different traffic conditions have been identified for the freeway system based on a suitable approximation of the steady-state speed-density relationship (as described in Section 2).

One $\mathrm{RH}$ regulator is associated with each traffic condition. More specifically, the considered transition cost function is:

$$
\begin{aligned}
h^{i}(k)=\sum_{j=1}^{S}\left(\xi_{j}(k)\right)^{2}+ & \left(\nu_{j}(k)\right)^{2} \\
& \\
k & =0,1, \ldots, K_{i}-1
\end{aligned}
$$

where $\xi_{j}$ and $\nu_{j}$ have the same meaning as in Section 2 and $K_{i}$ is a suitably chosen time horizon. It is to be noted that $K_{i}$ increases when $i$ becomes larger ( $\left.K_{i}<K_{i+1}, i=1, \ldots, N-1\right)$ since it is important to have a larger time horizon when the traffic density increases and possibly brings the system to congestion situations. Moreover, the final cost to be included in the finite horizon cost function relevant to the considered RH regulators is always characterized by $a^{i r}=1$ and $P^{i r}$ equal to the identity matrix.

So, $N$ different pairs model/regulator are assigned to the considered freeway portion and, in the overall control scheme, the supervisor, depending on the present system state and traffic conditions chooses the one to be applied to the freeway sections. The stabilizing properties of the adopted regulators have been stated in Section 3. It is still important to analyze the stabilizing properties of the overall control scheme. To this end, previous works have assessed that, by suitably defining switching rules to be adopted at any switching instant, some suitable stability properties can be ensured. Apart from classical Lyapunov stability which can be ensured by adopting very restrictive switching rules, the concept of $\epsilon$-practical stability has been defined in (Franco et al., 2004a), which turns out to be particularly suitable for the considered kind of systems.

Let us briefly recall the definition of $\epsilon$-practical stability.

Definition 4.1. $\epsilon$-Practical stability: Given $\epsilon>0$ and system (12) subject to a switching sequence $\Xi$ and a switching law $\mathcal{S}(\epsilon)$, the origin as an equilibrium point of system (12) is said to be $\epsilon$-practically stable under the action of the switching law $\mathcal{S}(\epsilon)$ if there exists $\delta=\delta(\epsilon)>0$ such that $\left\|x_{t}\right\|<\epsilon, t_{0}<t$ whenever $\left\|x_{t_{0}}\right\|<\delta$.

The meaning of the above definition is quite clear, what is required is that the norm of the state vector is always bounded by the pre-defined value $\epsilon$. Note that, in the present case, the system state is composed of the tracking errors between the freeway traffic density and traffic mean speed and the corresponding desired values. This means that guaranteeing $\epsilon$-practical stability ensures that the tracking errors are always bounded, which turns out to be a very significant property.

In (Franco et al., 2004a), several stability properties and, among them, $\epsilon$-practical stability, are carefully analyzed with reference to the proposed control scheme. In particular, the different stability properties are shown to be ensured under the application of suitable constraints to be fulfilled at each switching instant. Such constraints make use of the Lyapunov functions $\mathcal{V}^{i r}$ defined above and are not reported here for the sake of brevity. What is important to note here is that a switching law is defined in (Franco et $a l ., 2004 a$ ) guaranteeing that the origin as an equilibrium point of the considered system under the application of the proposed hybrid control scheme is $\epsilon-$ practically stable. By applying such a switching law, it is then possible to maintain bounded the defined tracking errors.

\section{SIMULATION RESULTS}

A real case study has been faced by the proposed control approach to test its effectiveness. The considered freeway portion consists of four two-lane sections, each of 1-km length, situated in the north-west part of the Italian freeway network. On-ramps and off-ramps are present in all the sections.

The analysis of real data led to the definition of $N=5$ different models and regulators for the considered freeway system. Each model is composed of equations (9) and (10). The approximated function $\tilde{V}\left(\rho_{j}(k)\right)$ has been calibrated on the basis of real data, finding the following desired values of traffic density $\tilde{\rho}_{1}=100[\mathrm{veh} / \mathrm{km}], j=$ $1, \ldots, S, \tilde{\rho}_{2}=150[\mathrm{veh} / \mathrm{km}], j=1, \ldots, S, \tilde{\rho}_{3}=$ $200[\mathrm{veh} / \mathrm{km}], j=1, \ldots, S, \tilde{\rho}_{4}=250[\operatorname{veh} / \mathrm{km}], j=$ $1, \ldots, S, \tilde{\rho}_{5}=300[\mathrm{veh} / \mathrm{km}], j=1, \ldots, S$. As regards the desired values of traffic mean speed: $\tilde{v}_{1}=$ $120[\mathrm{~km} / \mathrm{h}], j=1, \ldots, S, \tilde{v}_{2}=100[\mathrm{~km} / \mathrm{h}], j=$ $1, \ldots, S \quad \tilde{v}_{3}=80[\mathrm{~km} / \mathrm{h}], j=1, \ldots, S, \quad \tilde{v}_{4}=$ $60[\mathrm{~km} / \mathrm{h}], j=1, \ldots, S \quad \tilde{v}_{5}=40[\mathrm{~km} / \mathrm{h}], j=$ $1, \ldots, S$. The parameters of the freeway control objectives associated with the five regulators are: $K_{1}=5$, $K_{2}=8, K_{3}=K_{4}=K_{5}=10$.

The effectiveness of the proposed control scheme has been verified by means of an extensive simulation analysis. A case regarding the application of the hybrid controller in a case in which an accident affected the first section of the freeway portion is here reported (the section affected by the accident is the most downstream section of the considered freeway portion). Only few figures are shown for the sake of brevity. Specifically, some simulations are shown in Figs. 2 and 3 and compared with the recorded actual system behavior on the real freeway stretch.

\section{CONCLUSIONS}

A hybrid control scheme has been adopted for ramp metering of freeway systems. The proposed control 


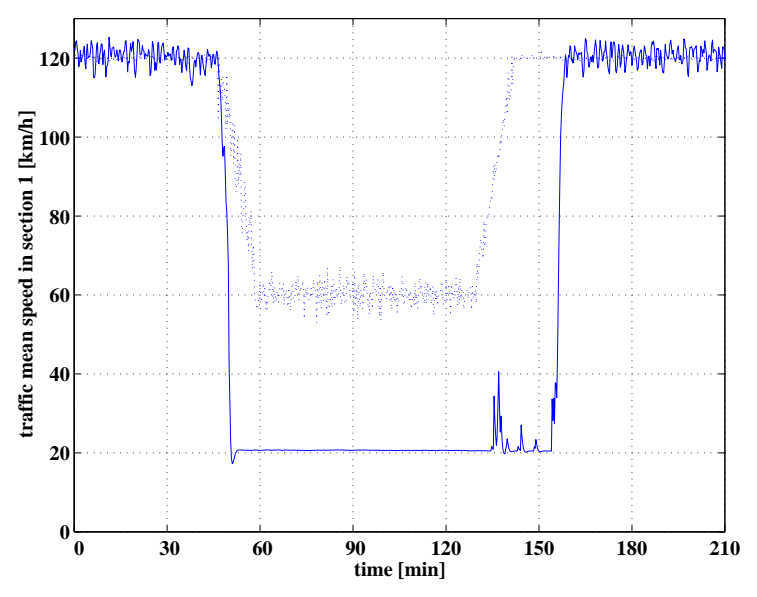

Fig. 2. Evolutions of the traffic mean speed in section 1 with (dotted line) and without (solid line) control.

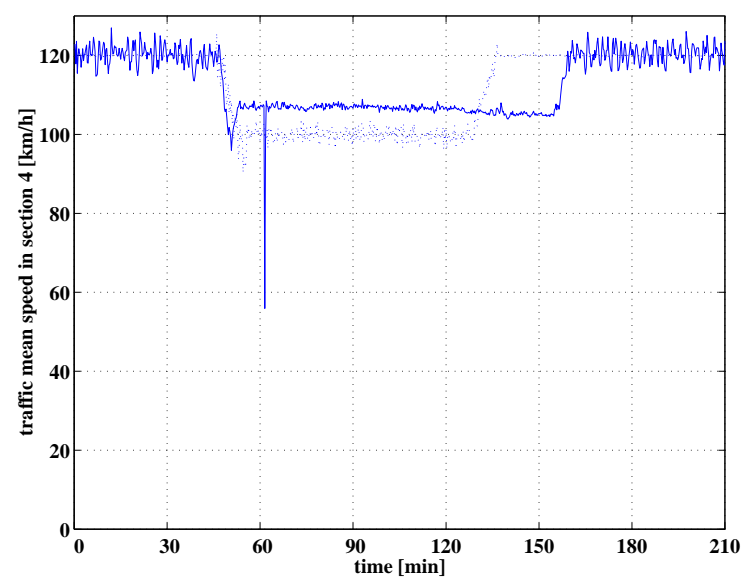

Fig. 3. Evolutions of the traffic mean speed in section 4 with (dotted line) and without (solid line) control.

scheme can guarantee several stability properties of the origin as an equilibrium point of the dynamic system representing the dynamics of tracking errors relevant to the traffic density and the traffic mean speed. Further research can regard the case in which the system state can be non fully measurable and the definition of a decentralized control scheme for wide freeway networks.

\section{REFERENCES}

Chien, Cheng-Chih, Y. Zhang and P.A. Ioannou (1997). Traffic density control for automated highway systems. Automatica 33(7), 1273-1285.

Di Febbraro, A., T. Parisini, S. Sacone and R. Zoppoli (2001). Neural approximations for feedback optimal control of freeway systems. IEEE Trans. on Vehicular Technology 50, 302-313.

Franco, E., S. Sacone and T. Parisini (2004a). Practically stable nonlinear receding-horizon control of multi-model systems. In: Proceedings of the IEEE Conference on Decision and Control. Paradise island, Bahamas.

Franco, E., S. Sacone and T. Parisini (2004b). Stable multi-model hybrid control of nonlinear systems. In: Proceedings of the American Control Confer- ence. Boston, MA.

Isaksen, L. and H. J. Payne (1973). Suboptimal control of linear systems by augmentation with application to freeway traffic regulation. IEEE Trans. Automatic Control 18, 210-219.

Keerthi, S. S. and E. G. Gilbert (1988). Optimal infinite-horizon feedback laws for a general class of constrained discrete-time systems: stability and moving-horizon approximations. Journal of Optimization Theory and Applications 57, 265-293.

Kotsialos, A., M. Papageorgiou and A. Messmer (2002). Coordinated and integrated control of motorway networks via nonlinear optimal control. Transportation Research 10C, 65-84.

Magliolo, M. and S. Sacone (2004). Validation of freeway traffic models on a real case study. Technical Report 2004/22. DIST.

Papageorgiou, M. and R. Mayr (1982). Optimal decomposition methods applied to motorway traffic control. International Journal of Control 35, 269280.

Papageorgiou, M., H. Hadj-Salem and J.M. Blosseville (1991). ALINEA: a local feedback control law for on-ramp metering. Transportation Research Record (1320), 58-64.

Papageorgiou, M., J.M. Blosseville and H. HadjSalem $(1990 a)$. Modelling and real-time control of traffic flow on the southern part of Boulevard Peripherique in Paris - part i: Modelling. Transportation Research A 24, 345-369.

Papageorgiou, M., J.M. Blosseville and H. HadjSalem (1990b). Modelling and real-time control on the southern part of Boulevard Peripherique in Paris - part II: Coordinated on-ramp metering. Transportation Research A 24, 361-370.

Parisini, T. and R. Zoppoli (1995). A receding-horizon regulator for nonlinear systems and a neural approximation. Automatica 31, 1443-1451.

Parisini, T. and S. Sacone (1999). A hybrid recedinghorizon control scheme for nonlinear systems. Systems and Control Letters 38, 187-196.

Parisini, T. and S. Sacone (2001). Stable hybrid control based on discrete-event automata and receding-horizon neural regulators. Automatica 37, 1279-1292.

Parisini, T., M. Sanguineti and R. Zoppoli (1998). Nonlinear stabilization by receding-horizon neural regulators. International Journal of Control 70, 3, 341-362.

Payne, H. J. (1971). Models of freeway traffic and control. In: Simulation Council Proc.. Vol. 1. pp. 5161.

Wattleworth, J.A. (1967). Peak period analysis and control of a freeway system. Highway Research Record 157, 1-10.

Zhang, H., S. Ritchie and W. Recker (1996). Some general results on the optimal ramp metering control problem. Transportation Research 4C, 51-69. 